\title{
Biodegradation of all-in-one self-etch adhesive systems at the resin-dentin interface
}

\author{
Sirichan CHIARAPUTT ${ }^{1,2}$, Pitchaya ROONGRUJIMEK ${ }^{2}$, Vanthana SATTABANASUK ${ }^{1}$, Nurot PANICH ${ }^{3}$, \\ Choltacha HARNIRATTISAI ${ }^{4}$ and Pisol SENAWONGSE ${ }^{4}$
}

\author{
${ }^{1}$ Department of Conservative Dentistry and Prosthodontics, Faculty of Dentistry, Srinakharinwirot University, Sukhumvit 23, Bangkok, Thailand 10110 \\ ${ }^{2}$ Dental Biomaterials Program, Faculty of Dentistry, Mahidol University, Yothe, Rajthevee Rd., Bangkok, Thailand 10400 \\ ${ }^{3}$ Faculty of Engineering, Chulalongkorn University, Phayathai Rd., Patumwan, Bangkok, Thailand 10330 \\ ${ }^{4}$ Department of Operative Dentistry, Faculty of Dentistry, Mahidol University, Yothe, Rajthevee Rd., Bangkok, Thailand 10400 \\ Corresponding author, Pisol SENAWONGSE; E-mail: dtpse@mahidol.ac.th
}

\begin{abstract}
This study evaluated the effects of two exogenous enzymes on the resin-dentin interface. Collagenase (Col) and acetylcholinesterase (Ach) were used to simulate salivary enzymes and accelerate the aging process of the bonding interfaces. Four adhesives, Adper Single Bond 2 (SB), Clearfil SE Bond (SE), Clearfil tri-S Bond (S3) and G-Bond (G), were bonded to the dentin surfaces. After storage in water with collagenase or acetylcholinesterase, the specimens were examined using a microtensile bond strength test (MTBS). Nanoleakage patterns were observed with a scanning electron microscope (SEM). The MTBS results demonstrated significantly lower bond strengths in the groups stored with either enzyme than in water. SB exhibited severe degradation after exposure to collagenase, while G showed severe degradation after exposure to acetylcholinesterase. All of the self-etch systems (SE, S3 and G) exhibited water-tree patterns within the adhesive layer when immersed in water for three months. The etch-and-rinse system (SB) showed nanoleakage within the hybrid layer and the adhesive.
\end{abstract}

Keywords: Interface, Self-etch adhesive, Bond strength, Nanoleakage

\section{INTRODUCTION}

Dentin bonding systems have become widely used in response to the increasing demand for esthetic dentistry. Many new generations of dentin bonding agents have been developed to overcome the flaws associated with the previous generations. Due to material handling problems, many studies have focused on simplified versions of dentin bonding systems. Recently, 'all-in-one' bonding system, also referred to as one-step self-etch adhesive system, has been developed ${ }^{1,2)}$. However, research has shown that such a system cannot achieve the same outcome as a three-step etch-and-rinse bonding system $^{3)}$. The etch-and-rinse technique is the most effective approach for achieving efficient and stable bonding to both enamel and dentin. The mechanism of etch-and-rinse adhesive to dentin is primarily diffusionbased and depends on the hybridization or infiltration of resin into the exposed collagen fibril network, which should be as complete as possible ${ }^{4)}$. In contrast, a self-etch system combines the etching and priming steps into one. Additionally, the bonding mechanism of self-etch systems differs from that of the etch-and-rinse system. The acidic primer in the self-etch adhesive system has been reported to remove or dissolve the smear layer, demineralize the underlying dentin, and simultaneously create a hybrid layer ${ }^{5)}$.

There are some differences among the self-etch materials as well. One study has shown that the performance of self-etch adhesive systems depends on the compositions of the materials used ${ }^{6}$. The clinical performance of the adhesives is limited by their bond durability. Excellent clinical performance has been reported for Clearfil SE Bond, a two-step self-etch adhesive $^{7}$. This high performance may result in part from the simultaneous demineralization and infiltration of dentin, which provides a shallow but uniform resininfiltrated dentin layer ${ }^{8}$. An effective approach to the clinical durability of adhesive materials may be necessary to improve the stability between the bond and the tooth ${ }^{9,10)}$. Factors that influence durability of bonding interfaces include time, temperature, and chemical interactions. Therefore, researchers have proposed various methods to test durability ${ }^{11}$.

Comparative studies have examined many aspects of these materials. For example, the microtensile bond strength test (MTBS) is commonly used to evaluate the effectiveness of the bond between the material and the tooth. One study has shown that the microtensile bond strength of self-etch adhesives gradually decreases over time ${ }^{12)}$. Transmission Electron Microscope (TEM) analysis has shown that this change is associated with an increase in nanoleakage ${ }^{13)}$, a change in the collagen fiber pattern over time ${ }^{14}$ and the 'water tree phenomenon' caused by bond failures in these adhesives ${ }^{15}$. However, there is still no evidence to indicate that nanoleakage or the water tree phenomenon of the etch-and-rinse system can weaken the bond interfaces over time. Such challenging monomer-collagen interactions might be the principal reason for what has been documented as nanoleakage.

Nanoleakage is a term that describes the diffusion of small ions or molecules within the hybrid layer in the absence of gap formation. Tracer molecules, such as 
silver nitrate, which are detectable by both SEM and TEM, are used to evaluate the quality of seal of the hybrid layer ${ }^{16)}$. It is important to determine the location and morphology of these nanometer-sized porosities because they may permit the hydrolysis of collagen fibers and the degradation of adhesive monomers. One study examined nanoleakage in the hybrid layer using a silver nitrate staining technique ${ }^{17)}$. Adhesive-dentin sandwiches, which were immersed in a silver nitrate solution, were prepared for SEM and TEM examinations using both the Clearfil Liner Bond and All-Bond 2 adhesive systems. Silver accumulation within the hybrid layers was demonstrated in both systems. The Clearfil Liner Bond system showed scattered silver particles in the bottom two thirds of the hybrid layer by both SEM and TEM, whereas All-Bond 2 revealed stained fiber-like structures within the full thickness of the hybrid layer ${ }^{17}$.

A study of biological degradation has provided information on degradation of bonding resin at the resin-dentin interface. This study revealed factors that influence nanoleakage at the resin-dentin interface ${ }^{18)}$.
Armstrong et al. reported significantly weaker bond strengths of resin-dentin interfaces after 12 weeks of storage in cholesterol esterase ${ }^{19)}$. It has also been demonstrated that hydrophilic dentin adhesives deteriorate over time. Exposed collagen matrices from acid-etched dentin, which had incompletely infiltrated collagen fibrils, were also susceptible to degradation over 500 days $^{20)}$. This finding emphasized the effect of collagenase at the resin-dentin interface.

The purpose of this study was to evaluate nanoleakage patterns and the microtensile strength bond to dentin of an etch-and-rinse, a two-step self-etch and an all-in-one adhesives after three months storage in different storage media.

\section{MATERIALS AND METHODS}

Thirty-two extracted, intact, non-carious human molars were used in this study. All of the teeth were impacted or non-occluded molars, which had been removed for clinical reasons and did not possess any code or identifier

Table 1 Composition and application techniques of bonding systems

\begin{tabular}{llll}
\hline Material & adhesive/pH & Manufacturer/Composition & Bonding procedure \\
\hline $\begin{array}{l}\text { Single Bond } 2 \\
\text { (SB) }\end{array}$ & Total-etching & 3M ESPE, St.Paul, MN, USA/ & 1. Apply Scotchbond etchant to dentin. \\
Batch no. & & Etchant: 37\% phosphoric acid & 2. Wait 15 seconds. Rinse for 10 seconds. \\
20080711 & & Adhesive:HEMA, bis-GMA, & 3. Blot excess water using cotton pellets or \\
& DMA's, methacrylate functional & mini-sponges until the surface appears \\
& Copolymer of polyacrylic and & glistening without pooling of water. \\
& polyitaconic acids, water, & 4. Apply 2-3 consecutive coats of adhesive \\
& ethanol, photo-initiator & immediately after blotting to the etched dentin \\
& & for 15 seconds with gentle agitation using a \\
& & fully saturated applicator.
\end{tabular}

Clearfil SE Bond Two-step (SE) self-etching

Batch no. 81154 pH 2
Clearfil tri-S

Bond

(S3)
Batch no. 51215
Kuraray, Tokyo, Japan/ Primer:10-MDP, HEMA, hydrophilic DMA, tertiary amine, water, photo-initiator Bond: 10-MDP, HEMA, bis-GMA, hydrophilic DMA, tertiary amine, silanated colloidal silica, photo-initiator
1. Apply primer to the entire surface with a sponge or a disposable brush tip.

2. Leave it in place for 20 seconds

3. Evaporate the volatile ingredients with a mild oil-free stream air

4. Apply bonding agent to the entire surface of the cavity with a sponge or a disposable brush tip.

5. After application, make the bond film as uniform as possible, using a gentle oil-free air stream.

6. Light-cure the adhesive for 10 seconds.

1. Apply bonding agent to the dentin surface.

2. Leave for 20 seconds followed by a strong air blow.

3. Light cure for 10 seconds.

1. Brush apply one coat of adhesive to the dentin

2. Wait 10 seconds.

3. Thin with high pressure syringe air.

4. Light cure for 10 seconds.
4-MET, UDMA, phosphate monomer, DMA component, fumed silica filler, acetone, water, photo-initiator 
that could be associated with any individual. The teeth were stored in a $0.5 \%$ thymol solution and used within three months of extraction. Flat dentin surfaces were produced by removing the coronal enamel of each tooth with a slow-speed diamond saw (Isomet; Buehler Ltd, Lake Bluff, IL, USA). The dentin surfaces were then polished with 600-grit silicon carbide ( $\mathrm{SiC}$ ) abrasive paper for 30 seconds under continuous water cooling to produce a standardized smear layer ${ }^{21}$.

\section{Specimen preparation}

The dentin surfaces were bonded to one of the four adhesives and resin composite according to the manufacturers' instructions, as shown in Table 1. Each bonding system was applied to the flat dentin surfaces of eight teeth. A nanofill resin composite (Z350; 3M ESPE, St. Paul, MN, USA) was built up incrementally on the bonded surface to a height of $4 \mathrm{~mm}$. The resin composite was then cured for 40 seconds after each 2-mm increment. A halogen light-curing unit (Elipar 2500; 3M ESPE) was used throughout the study. After immersion in water for 24 hours at $37^{\circ} \mathrm{C}$, the bonded teeth were serially sectioned perpendicularly to the adhesive interface in both the $\mathrm{X}$ and Y directions with a slow-speed diamond saw, which produced several beam-shaped specimens with crosssectional areas of approximately $1 \mathrm{~mm}^{2}$.

After sectioning, the specimens from each of the four bonding systems were further divided into four groups of 15 beams each. The specimens in the first three groups were kept in three different storage media: water, collagenase (Sigma-Aldrich, St. Louis, MO, USA) solution with an enzyme activity of 25 units per $\mathrm{mL}$ of storage solution, or acetylcholinesterase (Sigma-Aldrich) solution with an enzyme activity of 1 unit per $\mathrm{mL}$ of storage solution ${ }^{22,23)}$. The specimens were stored at $37^{\circ} \mathrm{C}$, and the media were changed every two days over a period of three months. The specimens in the fourth group were not continually stored but were instead immediately processed for the bond strength test.

\section{Microtensile bond strength test}

The microtensile bond strength test was performed after the teeth were stored in water for 24 hours, referred to as the initial bond strength, and after storage in either both collagenase and acetylcholinesterase for three months or water for three months. The specimens were fixed by their ends to a microtensile bond testing device with a cyanoacrylate adhesive (Model Repair II Blue; Sankin Industry Co., Ltd, Tokyo, Japan). They were tension tested at a crosshead speed of $0.5 \mathrm{~mm} / \mathrm{min}$ using a universal testing machine (Lloyd LF Plus Digital Testing Machine; Lloyd Instruments, Fareham, Hants, UK) until failure. The bond strength at the breaking point was automatically recorded in MPa by a microcomputer attached to the testing machine.

\section{Statistical analyses}

The means and standard deviations of the microtensile bond strengths were calculated for all sixteen groups. The Kolmogorov-Smirnov test (K-S test) was used with a cut-off of $\alpha=0.05$ to test the distribution of the data. Analysis of variance was performed to evaluate the possible effects of the type of storage medium relative to each storage time. Non-parametric statistical tests were used to evaluate the effect of each storage condition.

\section{Analysis of the fracture modes}

After the microtensile bond strength test, the fractured surfaces of both dentin side and resin side were investigated using a scanning electron microscope (SEM) according to the protocol reported by Senawongse et $a l{ }^{24)}$. Classification of the failure mode was determined by calculating each fractured pattern; i.e., adhesive failure, cohesive failure in resin, and cohesive failure in dentin, on the surface area as a percentage.

\section{SEM observation of the resin-dentin interface}

Two additional teeth were employed for each group. The teeth were prepared using the protocol for the microtensile bond test, as previously described. The beams were immersed in silver nitrate solution for 24 hours, developed in X-ray developing solution for 15 minutes and fixed for 2 minutes. After 24 hours, the specimens were embedded in epoxy resin. Then, they were polished with 600-, 800-, 1,000-, 1,200-, 2,500- and 4,000-grit $\mathrm{SiC}$ abrasive papers, followed by $6-\mu \mathrm{m}, 3-\mu \mathrm{m}$, $1-\mu \mathrm{m}$ and $0.3-\mu \mathrm{m}$ diamond polishing pastes (MetaDi; Buehler, Dusseldorf, Germany). The embedded specimens were then cleansed with an ultrasonic cleaner for 10 minutes and left in a desiccator for 24 hours. Before SEM analysis, the specimens were coated with approximately 10-nm thick carbon using a carbon coating machine (Hitachi E1010; Hitachi, Japan). The coated specimens were analyzed under SEM (Hitachi S3400-N; Hitachi) with magnifications of 5,000× and 10,000×.

\section{RESULTS}

\section{Microtensile bond strength test}

The results demonstrated the normal distribution of the data for all of the groups ( $p>0.05)$ except Clearfil tri-S Bond in collagenase (S3/Col), G-Bond at 24 hours (G/ initial) and G-Bond in acetylcholinesterase (G/Ach). The homogeneity of variance was analyzed using Levene's test. This test showed a significant difference at $p<0.01$. Therefore, the non-parametric statistic was subjected to further analysis. The Kruskal-Wallis test, selected to test the influence of each condition, showed that there was a significant difference among the tested groups. The Mann-Whitney test was used to test the differences among each pair of tested groups. The cut-off for statistical difference was set at $\alpha=0.0008$.

After 24 hours of storage, there were significant differences among the four bonding systems. The bond strengths of Adper Single Bond 2 at 24 hours (SB/initial), Clearfil SE Bond at 24 hours (SE/initial) and Clearfil tri-S Bond (S3/initial) groups were significantly higher than those of the G-Bond at 24 hours (G/initial) group. However, there were no significant differences in bond strengths between the SB, SE and S3 groups at 24 hours. 
Table 2 The microtensile bond strength of tested groups (mean $\pm \mathrm{SD}$ ) in MPa unit, $n=15$ for all tested groups

\begin{tabular}{ccccc}
\hline & Initial & Col & Ach & $3 \mathrm{~m}$ \\
\hline SB & $49.19 \pm 15.06^{\mathrm{a}, \mathrm{h}}$ & $20.75 \pm 14.04^{\mathrm{c}, \mathrm{f}}$ & $25.29 \pm 13.57^{\mathrm{c}, \mathrm{f}}$ & $36.98 \pm 12.02^{\mathrm{c}, \mathrm{h}}$ \\
$\mathrm{SE}$ & $49.52 \pm 14.98^{\mathrm{a}, \mathrm{d}}$ & $27.39 \pm 10.54^{\mathrm{c}, \mathrm{d}}$ & $25.55 \pm 17.95^{\mathrm{c}, \mathrm{d}}$ & $38.88 \pm 20.70^{\mathrm{c}, \mathrm{d}}$ \\
S3 & $39.33 \pm 11.95^{\mathrm{a}, \mathrm{g}}$ & $22.14 \pm 12.98^{\mathrm{c}, \mathrm{g}}$ & $19.82 \pm 11.09^{\mathrm{c}, \mathrm{e}}$ & $36.71 \pm 12.40^{\mathrm{c}, \mathrm{g}}$ \\
$\mathrm{G}$ & $29.31 \pm 6.46^{\mathrm{b}}$ & $23.37 \pm 8.33^{\mathrm{b}, \mathrm{c}}$ & $20.84 \pm 8.02^{\mathrm{b}, \mathrm{c}}$ & $29.15 \pm 12.18^{\mathrm{b}, \mathrm{c}}$ \\
\hline
\end{tabular}

The superscript indicates the statistical interactions between groups. Same superscript letters indicate no statistically significant differences $(p \geq 0.05)$.

Fracture Analysis of Microtensile Specimens

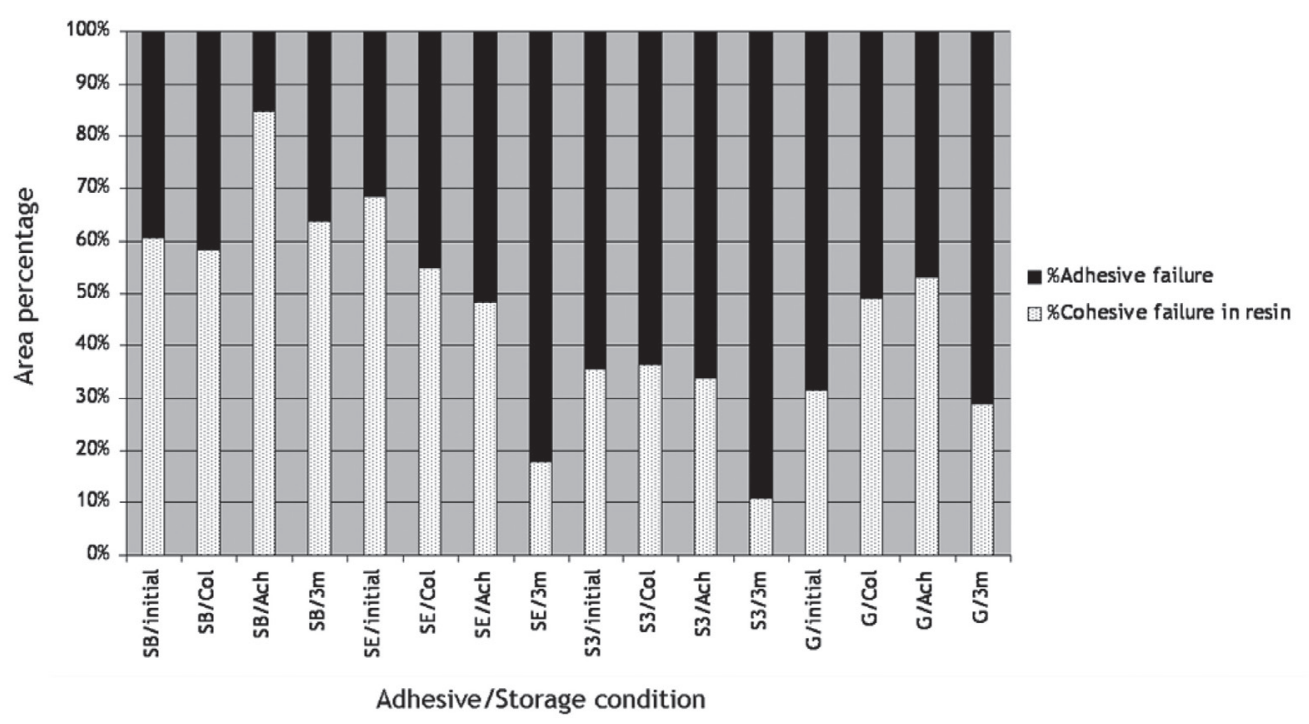

Fig. 1 The result of fracture analysis of microtensile specimens from resin and dentin fractured surface. No cohesive failure in dentin was found in all tested groups.

For other media at three-month storage time, statistically significant similarities of bond strengths among the four bonding systems were detected (Table 2).

There were significant differences in the bond strengths between the four storage conditions for each adhesive. In the SB group, the bond strength at 24 hours (SB/initial) was significantly higher than in the collagenase $(\mathrm{SB} / \mathrm{Col})$ or acetylcholinesterase groups $(\mathrm{SB} /$ Ach). However, there was no significant difference between the SB/initial and three-month water storage groups $(\mathrm{SB} / 3 \mathrm{~m})$. In the $\mathrm{S} 3$ group, there was significantly lower bond strength only in the acetylcholinesterase specimens (S3/Ach). For SE and G groups, no significant difference between the storage conditions was detected.

\section{Analysis of the fracture modes}

The results are shown in Fig. 1 with no cohesive failure in dentin was detected in all groups. In the SB group, cohesive failure in resin was found in a higher percentage area in the acetylcholinesterase group than in other storage conditions. In the SE, S3, and G groups, adhesive failure was found in a higher percentage area in three-month water storage than other storage media.

\section{SEM observation of the resin-dentin interface}

The SEM micrographs of each group are shown in Figs. 2 to 5 . In the SB group, the micrographs showed evidence of nanoleakage in some areas, such as the adhesive layer, the hybrid layer and the bottom of the resin tag. The thickness of the hybrid layer was approximately 4 to 6 $\mu \mathrm{m}$. In the $\mathrm{SB} / \mathrm{Col}$ interface (Figs. $2 \mathrm{c}$ and $2 \mathrm{~d}$ ), there was a large amount of nanoleakage at the top and bottom of the hybrid layer and throughout parts of the hybrid layer. Silver staining was also observed surrounding the resin tags. In the SE group, nanoleakage occurred in some areas at the bottom of the hybrid layer. The thickness of the hybrid layer was approximately 1 to 2 $\mu \mathrm{m}$. The bonding interface of the SE/Col showed a hybrid layer with silver staining at all depths. In the SE/Ach interface (Figs. 3e and 3f), a hybrid layer with a thickness of approximately 1 to $2 \mu \mathrm{m}$ was observed. The silver impregnation patterns were similar to those found in the 

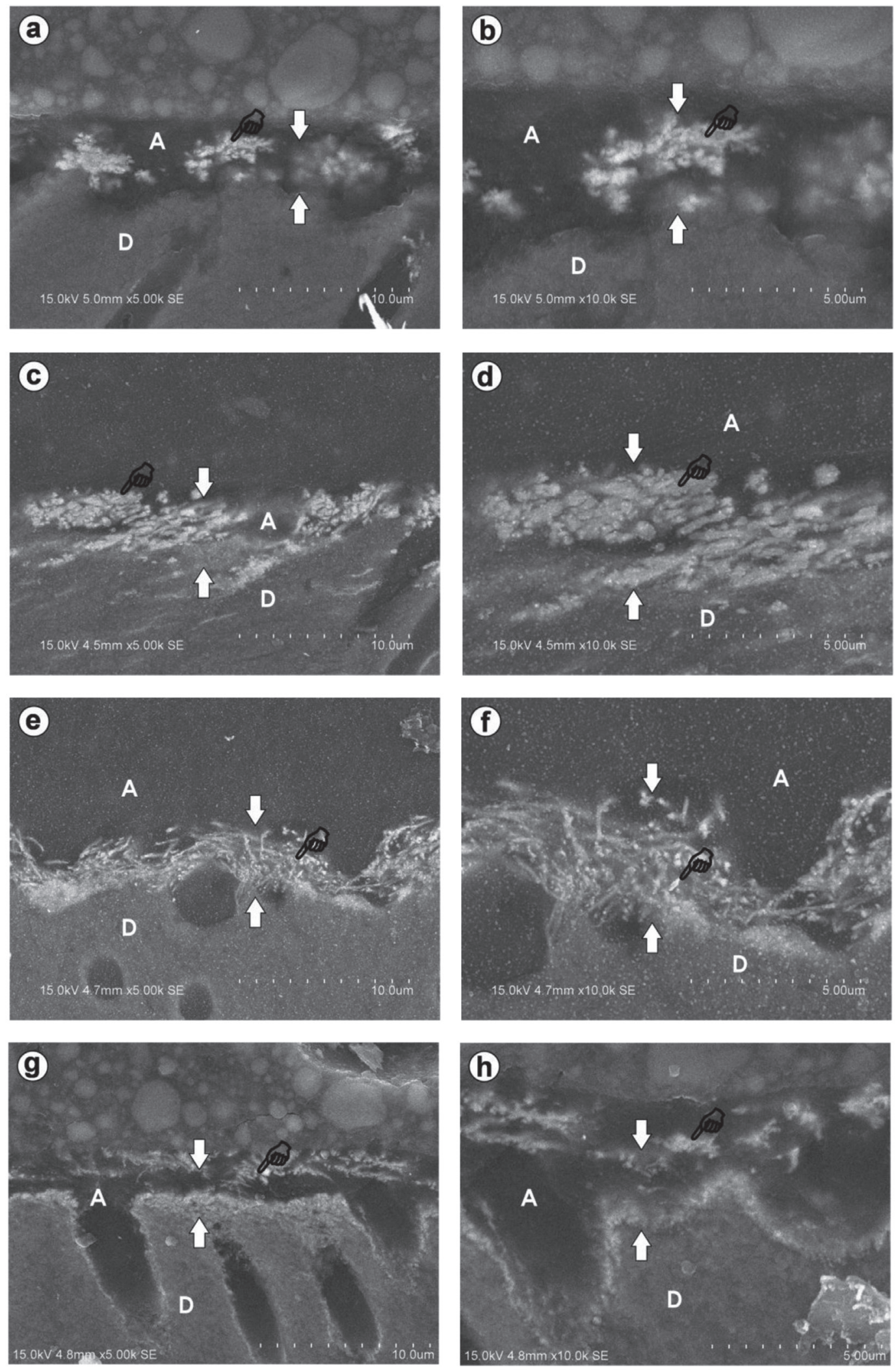

Fig. 2 The interface of SB specimens from SEM observation at the magnification 5,000× (left) and 10,000× (right), at $24 \mathrm{~h}$ $(\mathrm{a}, \mathrm{b})$, in collagenase for 3 months (c,d), in acetylcholinesterase for 3 months (e,f), and in water for 3 months (g,h). $\mathrm{A}=$ Adhesive and $\mathrm{D}=$ Dentin. The arrows indicate the thickness of the hybrid layer and the pointers indicate the silver penetration area or nanoleakage. 

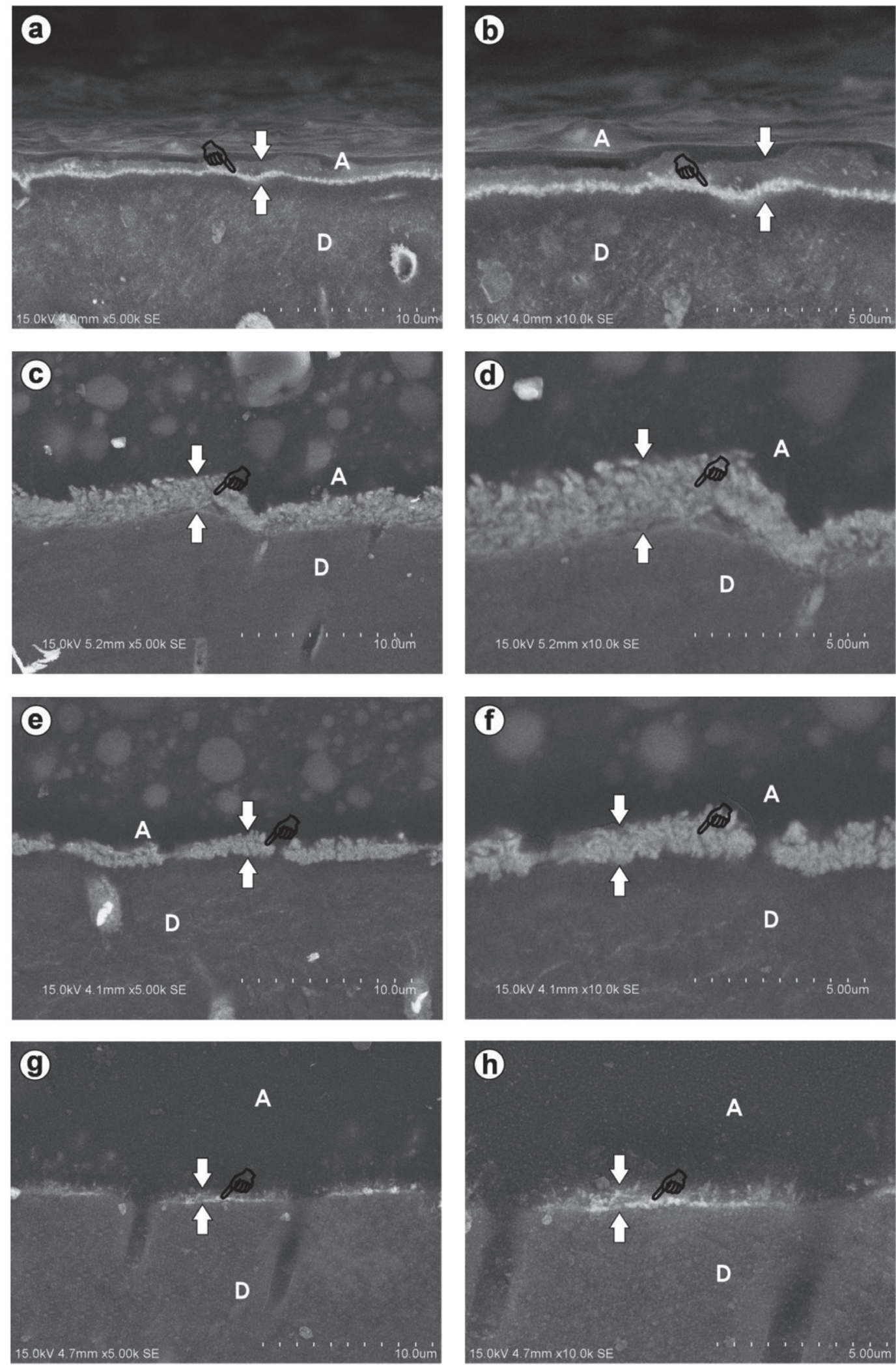

Fig. 3 The interface of SE specimens from SEM observation at the magnification 5,000× (left) and 10,000× (right), at $24 \mathrm{~h}$ (a,b), in collagenase for 3 months (c,d), in acetylcholinesterase for 3 months (e,f), and in water for 3 months (g,h), $\mathrm{A}=$ Adhesive and $\mathrm{D}=$ Dentin. The arrows indicate the thickness of the hybrid layer and the pointers indicate the silver penetration area or nanoleakage. 

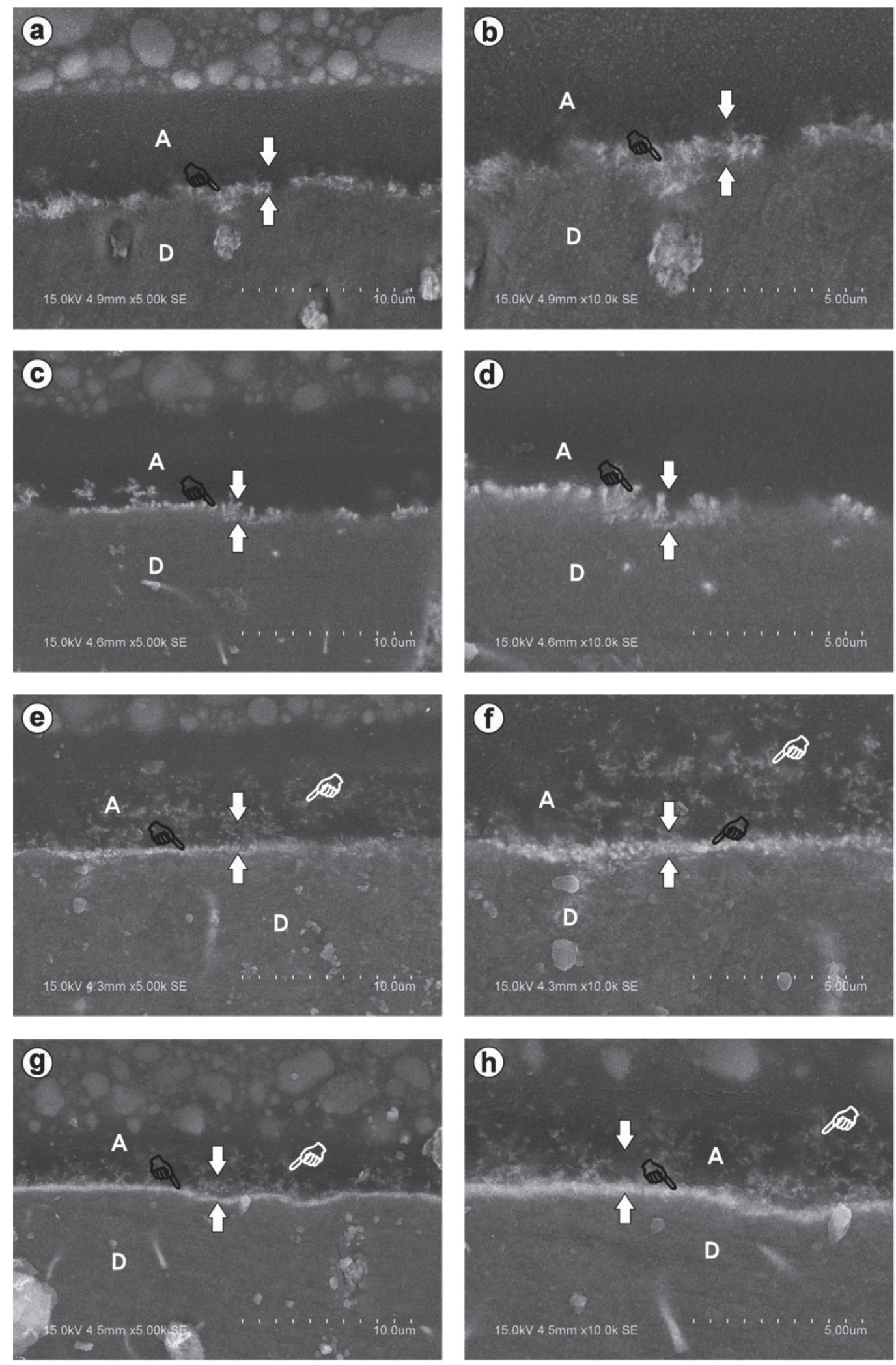

Fig. 4 The interface of S3 specimens from SEM observation at the magnification 5,000× (left) and 10,000× (right), at $24 \mathrm{~h}$ $(\mathrm{a}, \mathrm{b})$, in collagenase for 3 months (c,d), in acetylcholinesterase for 3 months (e,f), and in water for 3 months (g,h). $\mathrm{A}=$ Adhesive and $\mathrm{D}=$ Dentin. The arrows indicate the thickness of the hybrid layer and the pointers indicate the silver penetration area or nanoleakage. 

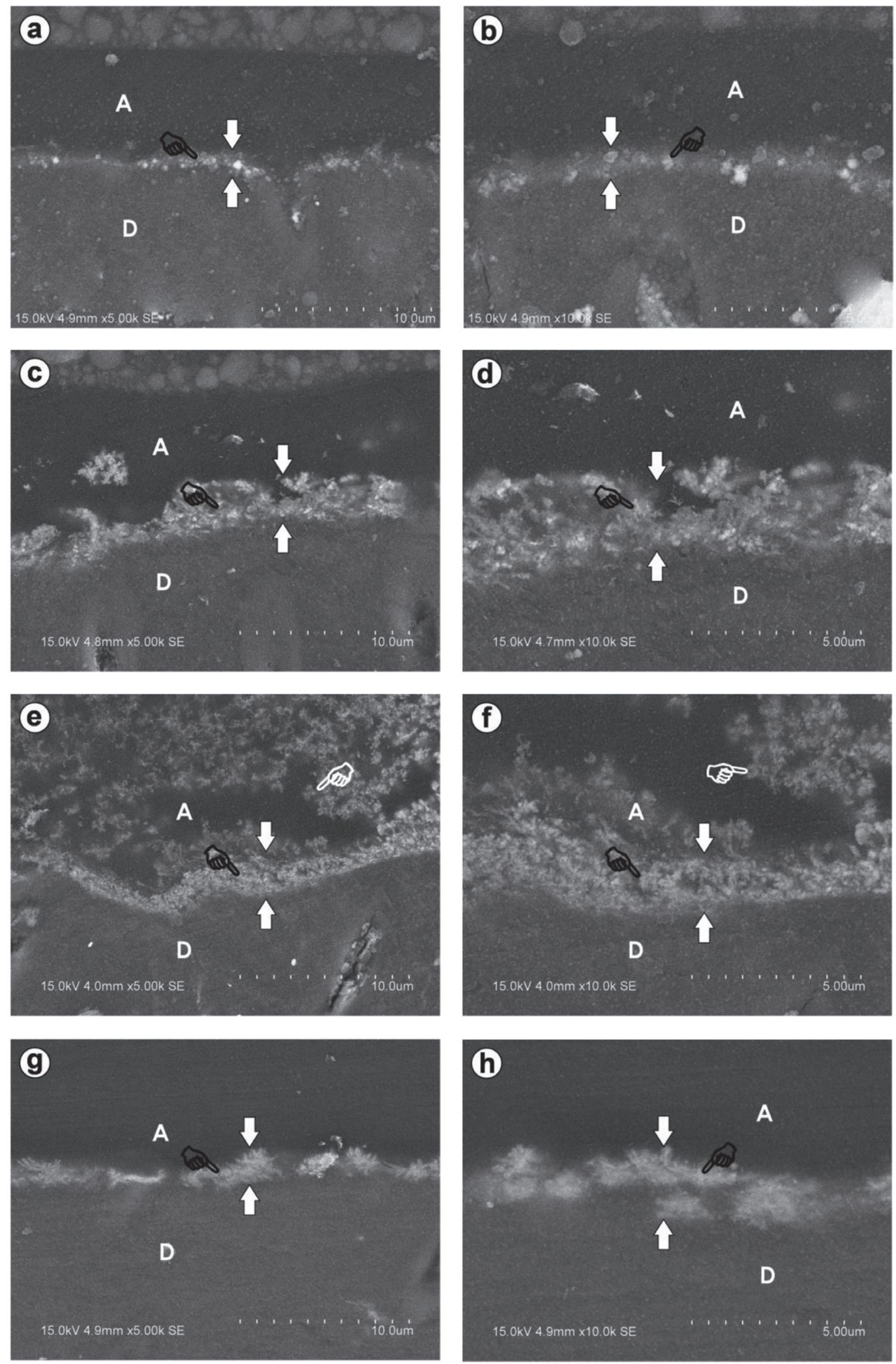

Fig. 5 The interface of G specimens from SEM observation at the magnification 5,000× (left) and 10,000× (right), at $24 \mathrm{~h}$ $(\mathrm{a}, \mathrm{b})$, in collagenase for 3 months (c,d), in acetylcholinesterase for 3 months (e,f), and in water for 3 months (g,h). $\mathrm{A}=$ Adhesive and $\mathrm{D}=$ Dentin. The arrows indicate the thickness of the hybrid layer and the pointers indicate the silver penetration area or nanoleakage. 
SE/Col interface (Figs. 3c and 3d). In the S3 group (Fig. 4), the hybrid layer was approximately 1 micron thick, and silver nitrate infiltration was found throughout the layer. In the $\mathrm{S} 3 / \mathrm{Col}$ interface (Figs. $4 \mathrm{c}$ and $4 \mathrm{~d}$ ), there was a large number of areas with silver deposits. Nanoleakage was found along the hybrid layer. The pattern of nanoleakage in the $\mathrm{S} 3 / \mathrm{Col}$ interface was similar to that in the S3/initial interface. Penetration of silver staining into the adhesive layer in a water tree pattern was observed. In the S3/Ach interface (Figs. 4e and 4f), nanoleakage was found along the hybrid layer and in the adhesive layer. In the G group (Fig. 5), nanoleakage was found within the hybrid layer. The thickness of the hybrid layer in this group was approximately $1 \mu \mathrm{m}$, and silver staining was localized to the hybrid layer. In the G/Col interface, nanoleakage was found throughout the thickness of the hybrid layer. Nanoleakage was also found in the porous region of the adhesive layer. The water tree appearance was also observed in some areas. In the G/Ach interface, nanoleakage was found along the hybrid layer and in the adhesive layer. A large amount of silver staining was observed within the hybrid layer and the adhesive layer. The staining found in this group was more than that in the S3 group under the same storage conditions. In the G/3m interface, nanoleakage was clearly observed in the hybrid layer. However, the pattern of silver nitrate infiltration was different from that in the 24-hour group. Figures $5 \mathrm{~g}$ and $5 \mathrm{~h}$ show a greater silver impregnation pattern in the $\mathrm{G} / 3 \mathrm{~m}$ group than in the 24-hour group.

\section{DISCUSSION}

The microtensile bond strength values for Adper Single Bond 2 (SB) and Clearfil SE Bond (SE) were greater than those for the other bonding systems tested in this study. These results are in agreement with those of previous studies $^{25,26)}$. Because SB is an etch-and-rinse adhesive system, the bonding mechanism of this adhesive is entirely different from that of the self-etch system. SB requires phosphoric acid to demineralize dentin layers that are more than five microns thick. However, the technique used for acid application also affects the quality of the demineralized area. SB can provide high bond strength but is quite technique sensitive ${ }^{27}$.

$\mathrm{SE}$ is a two-step self-etch system. This study showed that the thickness of the smear layer did not affect the bond strength in the self-etch bonding system. However, the acidity of the self-etch system did have some effect on the bond strength. SE considered to be a mild self-etch bonding system because it has a $\mathrm{pH}$ of more than 2.0. A previous study showed that this mild self-etch bonding system provided the highest bond strength compared to other tested self-etch systems ${ }^{25)}$.

Clearfil tri-S Bond (S3) and G-Bond (G) are one-step self-etch adhesive systems. The mean initial bond strength values for the $\mathrm{S} 3$ and $\mathrm{G}$ groups were $39.3 \mathrm{MPa}$ and $29.3 \mathrm{MPa}$, respectively, which were significantly different. The chemistry of the self-etch bonding system has a major effect on the performance of this system. S3 and $\mathrm{G}$ are both one-bottle mild self-etch bonding systems (all-in-one system); however, they are different in composition. G uses 4-META as the functional monomer, whereas S3 contains 10-MDP. A morphological study of resin-dentin interfaces by Perdigão et al. demonstrated a higher quality of the dentin-bonding interface of S3 compared to that of $\mathrm{G}^{28)}$. In a previous study, it was shown that some self-etch adhesive monomers, such as 10-MDP, could chemically adhere to hydroxyapatite and produce a stable bond ${ }^{29)}$. SE showed significantly higher bond strength than did G. In this study, S3 also provided significantly higher bond strength than did G at 24 hours. This result supported another study, which reported higher bond strengths for 10-MDP than for 4-META when forming a chemical bond to hydroxyapatite ${ }^{30)}$.

Although the microtensile bond strength was not different between the $\mathrm{SB} / \mathrm{Col}$ and $\mathrm{SB} / \mathrm{Ach}$ groups in the present study, the mode of failure was different. The SB/ Ach group showed a higher area percentage of cohesive failure in resin than did the $\mathrm{SB} / \mathrm{Col}$ group. It is possible that the chemical composition of the adhesive resin in the SB group is prone to degradation by acetylcholinesterase. A recent study by Shokati et al. reported that human-derived saliva esterase could degrade the Bis-GMA component of the Scotchbond three-step etch-and-rinse adhesive system ${ }^{31}$. Although the other two adhesive systems used in this study also contained Bis-GMA, the differences in the hybrid layer thickness and percentage composition could have produced the different results seen in this study. The SE and S3 showed high area percentages of adhesive failure when stored in water for three months. Although the bond strengths showed no significant differences in the SE group between all storage conditions, the higher adhesive failure in the three-month storage group could indicate a different fracture pattern. In the S3 group, significantly lower bond strength was found in the acetylcholinesterase group. However, the higher area percentage of adhesive failure could indicate a different fracture pattern from that observed in the other three groups. This phenomenon can be explained by the effect of water molecules throughout the interface of the stick-shaped specimens. It is known that the molecular weight of the exogenous enzyme is extremely high. Because the adhesive interface has been studied at the nanoleakage level, therefore, the size of the molecule must be bigger than that of the channel at the interface. The transportation of a substance along the interface can occur only if the substance is smaller than $1,000 \mathrm{~nm}$, the estimated diameter of a dentinal tubule. In addition, if the substance must work inside the collagen, it must be smaller than $100 \mathrm{~nm}$ to be able to penetrate the collagen fiber. Therefore, it is unlikely that highmolecular-weight exogenous enzymes could be more active than water or endogenous enzymes. An exogenous enzyme can penetrate into the interface only when there is a large enough gap at the interface ${ }^{32}$. However, in the SE and S3 groups, even the higher area percentage in the 3 -month group provided a higher bond strength than 
in those groups stored in exogenous enzyme. This result suggests that the exogenous enzymes used in this study truly affected the bond interface when the interface had an extremely small gap (nanoleakage). The effect of water in this study may have been overestimated due to the immersion of microtensile specimens in water because the water was able to diffuse through the dentinal tubule, resin composite and interface. However, the SB group was least affected by water when all the three-month specimens were compared. Our results support those of a study by Chiaraputt et al., who found that a more hydrophilic resin exhibited a greater reduction in flexural strength ${ }^{33)}$. Therefore, all self-etch bonding systems used in this study exhibited a greater percentage of adhesive failures after three months than did the etch-and-rinse bonding systems.

In the SEM study, the micrographs also showed different patterns of silver impregnation among the tested adhesive systems. This difference revealed the patterns of interaction between acidic monomers and dentin, which might have influenced the bond strength of these all-in-one adhesives. The different compositions of the one-bottle self-etch adhesives can produce different bond interfaces, even with similar acidity.

When immersed in collagenase, all of the tested adhesive systems showed different patterns of nanoleakage, though the bond strength values in collagenase for all of the tested groups did not differ significantly. The ability of the hybrid layer to absorb water and other small molecules is considered to be the cause of nanoleakage ${ }^{34)}$. Collagenase II was used in this study to represent salivary MMPs because the major MMPs in saliva are MMP-8 and MMP-9, which are found in collagenase II and gelatinase, respectively ${ }^{23,35)}$. It is known that degradation of the collagen in the hybrid layer can lead to bond failures over time ${ }^{36}$. However, the mechanism of this process is not yet clearly understood. In this study, the hybrid layer in the SB group displayed the most degradation during the three months of storage in collagenase. Sano ${ }^{37}$ who discovered nanoleakage, reported silver penetration into the hybrid layer even in the absence of gap formation. They reported that silver penetration could occur in a gap with a diameter of approximately 20 to $100 \mathrm{~nm}$. They explained that silver occupied nanometer-sized spaces around naked collagen fibrils where resin failed to infiltrate or where residual water had not been displaced by the adhesive resin. TEM analysis has demonstrated that water can pass from dentin and around resin tags to form water-filled channels that project from the hybrid layer into the overlying adhesive $^{37)}$. There was nanoleakage in the SB/ Col specimens along the hybrid layer, both horizontally and vertically. It can be assumed that only denuded collagen was attacked by the collagenase. It should be noted that the mechanism of bond formation is different between the etch-and-rinse and self-etch systems. The SB system, which represented the etch-and-rinse adhesive in this study, showed a large amount of nanoleakage. It was clear that the bonding interface of the etch-and-rinse system became more degraded under collagenase than did the self-etch system. This finding can be explained by the greater amount of collagen in the hybrid layer in the etch-and-rinse system than in the self-etch system. The etch-and-rinse system used in this study (SB) could not provide a perfect seal following demineralization by phosphoric acid. Therefore, a large amount of nanoleakage occurred. For the etch-and-rinse system, this phenomenon could be critical and might lead to post-operative sensitivity and even eventual bond failure. Hence, the nanoleakage pattern in the collagenase group could also indicate the quality of hybridization in the tested systems. The mildly acidic monomer in the self-etch system could only partially demineralize the dentin, which left hydroxyapatite for the chemical bond to resin bonding. Thus, the amount of denuded collagen and the thickness of the hybrid layer are less than what is seen in the etch-and-rinse system ${ }^{28)}$. The nanoleakage in self-etch adhesives can be explained by the performance of their hydrophilic monomers ${ }^{36)}$. The hydrophilic monomer in the self-etch adhesive can attract water after application. Thus, this monomer can cause the hybrid layer to become permeable to water.

This study provides a better understanding of the bonding mechanism and the areas susceptible to bond failures. The presence of nanoleakage in the oral cavity, where salivary MMPs can be found, makes restorations vulnerable to later degradation by MMPs. MMPs in the demineralized dentin and MMPs in the oral environment can both affect the bond interface. In patients who have an increased level of MMPs in the oral cavity, such as those with cancer, periodontitis or severe dental caries, special care is required to prevent the MMP-induced breakdown of the bonded restoration ${ }^{23,36)}$. In this study, all the tested systems yielded bond strength values in collagenase that were significantly lower than the initial bond strength values. This finding confirms the micrograph findings, which revealed greater nanoleakage than in the initial bond specimens.

Fracture analysis showed a lower percentage of cohesive failures in the collagenase group than in the other storage conditions for all tested bonding systems. This result confirms that collagenase can weaken the bond. Thus the percentage of adhesive failures is higher than that of cohesive failures in collagenase specimens of all tested adhesives.

Esterase has been reported to affect the degradation of resin-based materials ${ }^{21,38)}$. Previous study reported the effect of salivary esterase on biodegradation of dental composite $^{39}$. Human cholinesterase consists of acetylcholinesterase and pseudocholinesterase ${ }^{40)}$. Acetylcholinesterase was used in this study to investigate the biodegradation of resin at the interfaces. The chemistry of the resin also affected the amount of degradation in this study. The bond strength decreased with time for all adhesives when kept in acetylcholinesterase. SEM analysis provided additional details: the pattern of nanoleakage of $\mathrm{G}$ in acetylcholinesterase (G/Ach) was found within both the hybrid layer and the adhesive layer. Silver staining completely covered the adhesive layer of the $\mathrm{G}$ in acetylcholinesterase. S3 also exhibited 
nanoleakage in both the hybrid and adhesive layers. However, the nanoleakage in the adhesive layer in the S3 Bond was found to be less than that in the G group. SB and SE showed no nanoleakage in the adhesive layer. Both groups showed nanoleakage only in the hybrid layer, with different patterns. In the SB group, the nanoleakage was found along the hybrid layer at the area of encapsulated resin. This leakage pattern is due to the enzyme hydrolyzing the resin, leaving the collagen in the hybrid layer. Therefore, the nanoleakage in this pattern was similar to that in the collagen pattern in the hybrid layer. The nanoleakage pattern in the SE group was quite similar to that in the collagenase group (SE/Col). It is possible that the hybrid layer in this group contained both resin and denuded collagen in the same proportions. It was noted that only the resin of the adhesive layer in the all-in-one self-etch adhesives was hydrolyzed by acetylcholinesterase. Although the SE and the S3 groups had similar compositions, the $\mathrm{S} 3$ adhesive contains more water and hydrophilic resin, which can assist water diffusion from the dentin into the adhesive layer ${ }^{41}$. Therefore, the all-in-one self-etch adhesive systems may provide more channels for the enzyme to hydrolyze the adhesive which produced substantial nanoleakage patterns. Although the SB group exhibited some nanoleakage in the adhesive layer after 24 hours, this might reflect the voids left in the adhesive layer during the solvent evaporation process. However, the adhesive layer in the SB group did not show any signs of severe hydrolysis after three months of storage in acetylcholinesterase. In contrast, the hybrid layer in the SB group in acetylcholinesterase exhibited more nanoleakage than did the initial group. This phenomenon may be one reason that the breakdown of the interface is initiated from the hybrid layer. Prati et al. concluded that the permeability of the hybrid layer may make it vulnerable to degradation. Degradation at the hybrid layer can lead to the destruction of the interface ${ }^{42}$. Tay et al. reported that single-bottle adhesives behaved like permeable membranes after polymerization and were prone to degradation ${ }^{43}$. However, in this study, the SB group in acetylcholinesterase showed a different pattern of nanoleakage from that shown by the $\mathrm{S} 3$ and $\mathrm{G}$ groups. SB only exhibited severe degradation in the hybrid layer, whereas S3 and G exhibited more degradation in the adhesive layer. An oxygen-inhibited layer has been reported as a factor that can accelerate bond degradation. In a bonding system that has a high volume of water or solvent, it is possible to have areas of a thick oxygeninhibited layer. This oxygen-inhibited layer decreases the degree of polymerization of the bonding adhesive and also provides a susceptible area for hydrolysis. It has been reported that all-in-one self-etch bonding systems can create thicker oxygen-inhibited layers than can conventional bonding systems ${ }^{44)}$. A previous study indicated that human saliva contains esterase activities that can degrade resin-based materials, especially polymerized resin materials in the oral environment ${ }^{21}$. Therefore, any nanoleakage at the interface indicates that an adhesive restoration could have been exposed to esterase activities.

Water storage for three months was used to differentiate between the extrinsic and intrinsic factors affecting bond degradation. In this study, there was no significant difference in bond strength between 24 hours and three months in water for all tested groups. The micrographs for each group also supported the bond strength results. The nanoleakage found in the initial bond specimens and in the specimens stored for three months in water was less than that found in the collagenase and acetylcholinesterase specimens. Because the specimens were kept in each condition for three months, it is possible that both of the enzymes used in this study affected the bond interface, which caused more nanoleakage than was caused by three months of storage in water. The degradation of the bond interface after three months of storage was expected. The MMPs from dentin can also degrade the collagen at the hybrid layer, which causes more nanoleakage over time $e^{20,45-48)}$. The effects of moisture and bonding composition on bond degradation have been reported. A previous study suggested that the wet bonding technique may not be appropriate for the longevity of the bond when the adhesives contain HEMA ${ }^{49}$. Because water can cause poor polymerization of the hydrophilic resin, it is possible that some areas of the interface were vulnerable to degradation due to poor polymerization ${ }^{50)}$. In this study, the microtensile bond strengths decreased in all tested groups when immersed in water for three months. However, there were no significant differences from the initial bond strengths. The SEM micrographs showed different patterns of nanoleakage from those of the tested bonding systems. SB and S3 showed higher nanoleakage when immersed in water for three months than did the 24-hour specimens. S3 and G exhibited a water tree nanoleakage pattern along the adhesive layer. SE showed the least nanoleakage among the tested groups. All of the adhesives tested in this study contained HEMA except G. However, SE requires the hydrophobic resin to cover the primer layer. The application of hydrophobic resin can be the solution for any incomplete polymerization of resin in the primer. Although there were no significant differences in bond strengths in water among the tested groups, the nanoleakage patterns in the SEM micrographs provided basic knowledge about short-term bonding degradation. These bond interfaces should be studied over a longer period.

Although the results of the bond strength tests provided some basic information, the numerical values alone may not be enough to predict bond quality. In this study, all tested groups showed nanoleakage over time and in the presence of exogenous enzymes. The bond strength showed no significant difference from that of the initial bond after three months of storage in water. However, the pattern of nanoleakage showed some signs of bond degradation. Tagami et al. reported that the nanoleakage test and the morphological and chemical characteristics of the bonding interface had more potential to predict the bond durability than did the bond strength test ${ }^{511}$. 
Within the limitation of this study, it can be concluded that either enzymes used in this study can affect the bond interfaces of tested adhesives. The nanoleakage patterns of all-in-one self-etch adhesives (Clearfil tri-S Bond and G-Bond) were different from two-step self-etch (Clearfil SE Bond) and etch-and-rinse (Adper Single Bond 2) adhesives. This in vitro study, however, demonstrated biodegradation of the resin-dentin interface for three-month storage. Moreover, it is of interest to evaluate the long-term alteration of bond to clinically relevant substrates, e.g., caries-affected dentin, abraded/eroded dentin. Further study, therefore, is needed to observe the changes of bond not only in longer storage time but also in clinically related situations for better understanding the lifetime of resin-dentin bonds.

\section{ACKNOWLEDGMENTS}

The authors would like to thank the National Nanotechnology Center and the Research Unit, Faculty of Dentistry, Mahidol University for their support throughout the project. We would also like to express our thanks to Dr. M. Kevin O Carroll, Professor Emeritus of the University of Mississippi School of Dentistry, USA, and Faculty Consultant at Chiang Mai University Faculty of Dentistry, Thailand, for his assistance with the preparation of the manuscript.

\section{REFERENCES}

1) Prati C, Chersoni S, Mongiorgi R, Pashley DH. Resininfiltrated dentin layer formation of new bonding systems. Oper Dent 1998; 23: 185-194.

2) Prati C, Ferrieri P, Galloni C, Mongiorgi R, Davidson CL. Dentine permeability and bond quality as affected by new bonding systems. J Dent 1995; 23: 217-226.

3) Fritz UB, Finger WJ. Bonding efficiency of single-bottle enamel/dentin adhesives. Am J Dent 1999; 12: 277-282.

4) Nakabayashi N, Kojima K, Masuhara E. The promotion of adhesion by the infiltration of monomers into tooth substrates. J Biomed Mater Res 1982; 16: 265-273.

5) Jacques $P$, Hebling J. Effect of dentin conditioners on the microtensile bond strength of a conventional and a self-etching primer adhesive system. Dent Mater 2005; 21: 103-109.

6) Moura SK, Pelizzaro A, Dal Bianco K, de Goes MF, Loguercio $\mathrm{AD}$, Reis A, Grande RH. Does the acidity of self-etching primers affect bond strength and surface morphology of enamel. J Adhes Dent 2006; 8: 75-83.

7) Van Meerbeek B, Kanumilli PV, De Munck J, Van Landuyt K, Lambrechts P, Peumans M. A randomized, controlled trial evaluating the three-year clinical effectiveness of two etch \& rinse adhesives in cervical lesions. Oper Dent 2004; 29: 376-385.

8) Tay FR, Pashley DH. Aggressiveness of contemporary self-etching systems. I: Depth of penetration beyond dentin smear layers. Dent Mater 2001; 17: 296-308.

9) Breschi L, Mazzoni A, Nato F, Carrilho M, Visintini E, Tjaderhane L, Ruggeri A Jr, Tay FR, Dorigo Ede S, Pashley H. Chlorhexidine stabilizes the adhesive interface: a 2-year in vitro study. Dent Mater 2010; 26: 320-325.

10) Sadek FT, Castellan CS, Braga RR, Mai S, Tjaderhane L, Pashley DH, Tay FR. One-year stability of resin-dentin bonds created with a hydrophobic ethanol-wet bonding technique.
Dent Mater 2010; 26: 380-386.

11) De Munck J, Van Landuyt K, Peumans M, Poitevin A, Lambrechts P, Braem M, Van Meerbeek B. A critical review of the durability of adhesion to tooth tissue: methods and results. J Dent Res 2005; 84: 118-132.

12) Toledano M, Osorio R, Osorio E, Aguilera FS, Yamauti M, Pashley DH, Tay FR. Durability of resin-dentin bonds: effects of direct/indirect exposure and storage media. Dent Mater 2007; 23: 885-892.

13) Hashimoto M, De Munck J, Ito S, Sano H, Kaga M, Oguchi H, Van Meerbeek B, Pashley DH. In vitro effect of nanoleakage expression on resin-dentin bond strengths analyzed by microtensile bond test, SEM/EDX and TEM. Biomaterials 2004; 25: 5565-5574.

14) Reis AF, Giannini M, Pereira PN. Long-term TEM analysis of the nanoleakage patterns in resin-dentin interfaces produced by different bonding strategies. Dent Mater 2007; 23: 1164-1172.

15) Duarte S, Jr., Perdigao J, Lopes MM. Effect of dentin conditioning time on nanoleakage. Oper Dent 2006; 31: 500-511.

16) Sano H, Takatsu T, Ciucchi B, Horner JA, Matthews WG, Pashley DH. Nanoleakage: leakage within the hybrid layer. Oper Dent 1995; 20: 18-25.

17) Sano H, Yoshiyama M, Ebisu S, Burrow MF, Takatsu T, Ciucchi B, Pashley DH. Comparative SEM and TEM observations of nanoleakage within the hybrid layer. Oper Dent 1995; 20: 160-167.

18) Bortolotto T, Onisor I, Krejci I, Ferrari M, Tay FR, Bouillaguet S. Effect of cyclic loading under enzymatic activity on resin-dentin interfaces of two self-etching adhesives. Dent Mater 2008; 24: 178-184.

19) Armstrong SR, Jessop JL, Armstrong SR, Jessop JL, Vasgas MA, Zou Y, Qian F, Campbell JA, Pashley DH. Effects of exogenous collagenase and cholesterol esterase on the durability of the resin-dentin bond. J Adhes Dent 2006; 8: 151-160.

20) Hashimoto M, Tay FR, Ohno H, Sano H, Kaga M, Yiu C, Kumagai ,H. Kudou Y, Kubota M, Oguchi H. SEM and TEM analysis of water degradation of human dentinal collagen. $\mathrm{J}$ Biomed Mater Res B Appl Biomater 2003; 66: 287-298.

21) Lin BA, Jaffer F, Duff MD, Tang YW, Santerre JP. Identifying enzyme activities within human saliva which are relevant to dental resin composite biodegradation. Biomaterials 2005; 26: 4259-4264.

22) Uitto VJ, Suomalainen K, Sorsa T. Salivary collagenase. Origin, characteristics and relationship to periodontal health. J Periodontal Res 1990; 25: 135-142.

23) Hannas AR, Pereira JC, Granjeiro JM, Tjaderhane L. The role of matrix metalloproteinases in the oral environment. Acta Odontol Scand 2007; 65: 1-13.

24) Senawongse P, Srihanon A, Muangmingsuk A, Harnirattisai C. Effect of dentine smear layer on the performance of self-etching adhesive systems: A micro-tensile bond strength study. J Biomed Mater Res B Appl Biomater 2010; 94: 212-221.

25) Reis A, Grandi V, Carlotto L, Bortoli G, Patzlaff R, Rodrigues Accorinte M de L, Dourado Loguercio A. Effect of smear layer thickness and acidity of self-etching solutions on early and long-term bond strength to dentin. J Dent 2005; 33: 549-559.

26) Osorio R, Pisani-Proenca J, Erhardt MC, Osorio E, Aguilera FS, Tay FR, Toledano M. Resistance of ten contemporary adhesives to resin-dentine bond degradation. J Dent 2008; 36: 163-169.

27) Wang Y, Spencer P. Effect of acid etching time and technique on interfacial characteristics of the adhesive-dentin bond using differential staining. Eur J Oral Sci 2004; 112: 293-299.

28) Perdigão J, Lopes MM, Gomes G. In vitro bonding performance of self-etch adhesives: II —ultramorphological evaluation. 
Oper Dent 2008; 33: 534-549.

29) Moszner N, Salz U, Zimmermann J. Chemical aspects of self-etching enamel-dentin adhesives: a systematic review. Dent Mater 2005; 21: 895-910.

30) Yoshida Y, Nagakane K, Fukuda R, Nakayama Y, Okazaki M, Shintani H, Inoue S, Tagawa Y, Suzuki K, De Munck J, Van Meerbeek B. Comparative study on adhesive performance of functional monomers. J Dent Res 2004; 83: 454-458.

31) Shokati B, Tam LE, Santerre JP, Finer Y. Effect of salivary esterase on the integrity and fracture toughness of the dentin-resin interface. J Biomed Mater Res B Appl Biomater 2010; 94: 230-237.

32) Lin CP, Douglas WH, Erlandsen SL. Scanning electron microscopy of type I collagen at the dentin-enamel junction of human teeth. J Histochem Cytochem 1993; 41: 381-388.

33) Chiaraputt S, Mai S, Huffman BP, Kapur R, Agee KA, Yiu CK, Chan DC, Harnivattisai C, Arola DO, Rueggeberg FA, Pashley DH, Tay FR. Changes in resin-infiltrated dentin stiffness after water storage. J Dent Res 2008; 87: 655-660.

34) Chersoni S, Suppa P, Breschi L, Ferrari M, Tay FR, Pashley DH, Prati C. Water movement in the hybrid layer after different dentin treatments. Dent Mater 2004; 20: 796-803.

35) Fingleton B, Menon R, Carter KJ, Overstreet PD, Hachey DL, Matrisian LM, Mclntyre JO. Proteinase activity in human and murine saliva as a biomarker for proteinase inhibitor efficacy. Clin Cancer Res 2004; 10: 7865-7874.

36) Yiu CK, Tay FR, Pashley DH, King NM, Suh BI, Itthagarun A. Effect of resin hydrophilicity on tracer penetration. A preliminary study. Am J Dent 2005; 18: 160-164.

37) Sano H. Microtensile testing, nanoleakage, and biodegradation of resin-dentin bonds. J Dent Res 2006; 85: 11-14

38) Labow RS, Meek E, Santerre JP. Model systems to assess the destructive potential of human neutrophils and monocytederived macrophages during the acute and chronic phases of inflammation. J Biomed Mater Res 2001; 54: 189-197.

39) Finer Y, Santerre JP. Salivary esterase activity and its association with the biodegradation of dental composites. J Dent Res 2004; 83: 22-26.
40) Ryhänen R, Närhi M, Puhakainen E, Hänninen O, KontturiNärhi V. Pseudocholinesterase activity and its origin in human oral fluid. J Dent Res 1983; 62: 20-23.

41) Tay FR, Pashley DH, Suh BI, Hiraishi N, Yiu CK. Water treeing in simplified dentin adhesives —deja vu? Oper Dent 2005; 30: 561-579.

42) Prati C, Chersoni S, Acquaviva GL, Breschi L, Suppa P, Tay FR, Pashley DH. Permeability of marginal hybrid layers in composite restorations. Clin Oral Invest 2005; 9: 1-7.

43) Tay FR, Frankenberger R, Krejci I, Bouillaguet S, Pashley DH, Carvalho RM, Lai CN. Single-bottle adhesives behave as permeable membranes after polymerization. I. In vivo evidence. J Dent 2004; 32: 611-621.

44) Hashimoto M, Fujita S, Endo K, Ohno H. In vitro degradation of resin-dentin bonds with one-bottle self-etching adhesives. Eur J Oral Sci 2009; 117: 611-617.

45) Pashley DH, Tay FR, Yiu C, Hashimoto M, Breschi L, Carvalho RM, Ito S. Collagen degradation by host-derived enzymes during aging. J Dent Res 2004; 83: 216-221.

46) Carrilho MR, Tay FR, Pashley DH, Tjaderhane L, Carvalho RM. Mechanical stability of resin-dentin bond components. Dent Mater 2005; 21: 232-241.

47) Hebling J, Pashley DH, Tjaderhane L, Tay FR. Chlorhexidine arrests subclinical degradation of dentin hybrid layers in vivo. J Dent Res 2005; 84: 741-746.

48) Sulkala M, Tervahartiala T, Sorsa T, Larmas M, Salo T, Tjaderhane L. Matrix metalloproteinase-8 (MMP-8) is the major collagenase in human dentin. Arch Oral Biol 2007; 52: 121-127.

49) Reis A, Loguercio AD, Carvalho RM, Grande RH. Durability of resin dentin interfaces: effects of surface moisture and adhesive solvent component. Dent Mater 2004; 20: 669-676.

50) Jacobsen T, Soderholm KJ. Some effects of water on dentin bonding. Dent Mater 1995; 11: 132-136.

51) Tagami J, Nikaido T, Nakajima M, Shimada Y. Relationship between bond strength tests and other in vitro phenomena. Dent Mater 2010; 26: e94-e99. 